\title{
Perceptions And Challenges Of Healthcare Workers In Making Ethical Decisions Of Withholding And Withdrawing Life Support From Terminally III Patient In Tertiary Hospital, Mwanza Region- Northwestern Tanzania
}

NDAKIBAE GABRIEL MABEGA ( $\square$ ndakibaegabriel@gmail.com )

Mwanza College of Health and Allied Sciences https://orcid.org/0000-0001-5186-6689

Paschal Ruggajo

Muhimbili University of Health and Allied Sciences

Research article

Keywords: Withholding, Withdrawing, Life Support, Perceptions, Healthcare workers, Challenges

Posted Date: March 11th, 2020

DOI: https://doi.org/10.21203/rs.3.rs-16697/v1

License: (c) (i) This work is licensed under a Creative Commons Attribution 4.0 International License.

Read Full License 


\section{Abstract}

Background: Making ethical decisions in Withholding and withdrawing life support measures from terminally ill patients are challenges in clinical practice. Still there is no consensus around the world. In Tanzania, there is neither guideline nor legal frameworks governing the practice.

Methods: Aim: The aim of the study was to explore the perceptions and ethical challenges of healthcare workers in making ethical decisions of withholding and withdrawing life supportive measures from terminally ill patients.

Results: A healthcare workers perceptions varies in decisions of withholding and withdrawing life supportive measures from terminally ill patients.

Conclusions: Decisions of withholding and withdrawing life support measures from terminally ill patients face many challenges. Lack of legal and ethical framework to guide the practices inhibits the ethical decisions of physicians and nurses.

\section{Background}

Withholding and withdrawing life supportive measures from terminally ill patients occurs when interventions (e.g. mechanical ventilation, antibiotics, nutrition and hydration) are no longer beneficial to the patient's quality of life.The majority of deaths occur in the intensive care unit (ICU) after withdrawal and withholding life supportive measures. In many western hospitals, withholding and withdrawing life support from terminally ill patients in the ICU is a practice that occurs when no other treatments are deemed beneficial for a dying patient.Every day, a patient might die in the ICU and many ICUs have specific policies and guidelines that health care professionals can follow regarding withholding and withdrawing of life supportive measures. However, due to different cultural, religious, and other beliefs among healthcare workers, perceptions on withholding and withdrawal of life support measures from terminally ill patients may vary ${ }^{2}$.The ultimate goal of health care professionals is to alleviate pain, suffering and to improve the quality of life of their patients; withdrawing and withholding of life supportive measures may be perceived as contrary to providers $₫$ professional and moral obligations.

There are no studies that explore the perceptions and challenges of healthcare workers in end of life decisions in Tanzanian ICUs However, withholding and withdrawal life support measures is not legally authorized but traditionally are quietly practiced by healthcare workers.

Therefore this study explored the perceptions and challenges of healthcare workers working in the adult intensive care unit (AICU) of Bugando Medical Centre toward withholding and withdrawing of life support measures in terminally ill patients. By understanding perceptions and challenges of healthcare workers, we can begin to develop guidelines that can be used in clinical practice. Thus, this study will help nurses and physicians, hospital managers, hospital administrators, policy makers and other stakeholders to have a clearer understanding of this important issue. 


\section{Methods}

\section{Study design.}

A qualitative descriptive (QD) study design were used to explore perceptions and ethical challenges of healthcare workers regarding the ethical decisions in withdrawing and withholding life support from terminally ill patients in the AICU. Purposive sampling was used to reach study participants.

\section{Study setting.}

The study was conducted in Adult Intensive Care Unit of Bugando Medical Centre (BMC). BMC is Zonal Tertiary hospital for Lake Zone Tanzania. It has a bed capacity of 950 beds. It receives referral from eight regions, that is Kigoma, Simiyu, Mwanza, Kagera, Tabora, Mara, Geita and Shinyanga The bed capacity for AICU is 13 beds and eight mechanical ventilators.

\section{Participant's characteristics.}

The study involved 19 respondents among 26 healthcare workers in AICU, nine were males and ten females, and among them five were physicians and fourteen nurses of different cadres, seven degree nurses and seven diploma nurses. The mean ages of the respondents were between 36 - 43 years. ICU and clinical experience were differs with respect to respondents, however majority of respondents range between 11-20 years and 3-10 years $\$ experience in clinical and ICU practice respectively.

\section{Data analysis.}

Data analysis used both inductive and deductive thematic analysis, themes were pre-determined from conceptual frame work and interview guide, some categories were emerged during data analysis. Data collection and analysis was done simultaneously by using thematic content analysis with the assistance of computer software, NVIVO. Field notes, audio, and field observations were prepared, producing a verbatim transcript of the interviews. Lists of all codes were given a name and concise definition to create a label segment of text (theme). All the data listed in the code book were labeled to identify all segments of the text, condensed and the codes were assigned to develop sub categories and categories and the themes was then identified from the codes, subcategories and categories.

\section{Ethical consideration.}

The proposal was submitted to Muhimbili University of health and Allied Sciences (MUHAS) ETHICAL COMMITTEE and granted an ethical certificate approval Ref. No DA.287/298/01A/. Also, the proposal was submitted to CUHAS/BMC RESERCH AND ETHICAL COMMITTEE and granted an ethical certificate approval number: No CREC/351/2019. Permission for conducting the research was also sought from the Director of Bugando Medical Centre.

\section{Results}


From the analysis of interview documents two main themes were emerged.

(1) Ethical challenges facing healthcare workers in making decisions of withholding and withdrawing life support from terminally ill

(2) Factors influence the decision of withholding and withdrawing life support measures from terminally ill patients.

\section{Dilemma in decision making.}

Data from interviews found that, healthcare workers in the ICU face many challenges in making decisions of withholding and withdrawing life support measures from terminally ill patients. They face many ethical dilemmas, especially when it comes to the point to decide whether to continue or stop interventions for terminally ill patients. However they have a diverse view in making decisions of withholding and withdrawing life support. In some cases healthcare workers might think that, patient condition can never be recovered but sometime when they continue interventions, the patient prognosis become well. Due to that, early withdrawing or withholding life support from terminally ill patients is somehow delayed. As one nurse participant noted below the concern that some patients might recover:

"... The patients you may think that they cannot be improved, but they improved, we indicated to stop continuous medication or all management, but when we continue management the patients recover. So we found that stopping early the interventions or whatever medication is dangerous, we decide to leave the nature to take place. There are some patients you expecting are going to die soon but they are not died, they recovered" (Nurse \# 11).

On the other hand, healthcare workers views to continue interventions for terminally ill patients who are ventilators dependent may be perceived as a misuse of resources and energy, because there is no chance of patient survival. Therefore, more effort is better directed to patients who have a high chance of recovery. As one of the nurse views in caring terminally ill patients in ICU.

"To me such patients who are seriously depend on the mechanical ventilators it is better to educate relative to remove from the machine rather than put on expenses which are painful in future" (Nurse \# 3)

Withholding and withdrawing life support from terminally ill patients was also seen in a different way to other participants. ICU services are very expensive, and the one who is responsible for payment is the patient himself or their relatives. If the patient stays on mechanical ventilator for a long time, the hospital expenditures becomes high. Therefore keeping the hopeless patient on a mechanical ventilator for a period of time may be beneficial to the hospital and not to the patient, because they generate income despite the patient condition. This was noted by a one participant below:

"...but for the hospital is a benefit because themselves are just using material and this material will be paid by relatives so actually for other side it is paining because sometime relatives use money for the 
patient who have no hope..."(Nurse \# 1).

In addition, the duration of patient to stay on a mechanical ventilator before decision is made, on withdrawing or withholding life support measures. It has been found that, there is no specific time for the terminally ill patient to stay in mechanical ventilator. Patient has to stay in mechanical ventilator until the nature takes place. The study has found that, once the ICU is full occupied there is no chance of receiving another patient. One participant stated the following:

"Actually, we do not stop ventilators because other patients are coming. If you stop the ventilator you are terminating the life of the patient. We wait until the nature takes place, but may be if anybody happens to die, we can continue to use the available ventilators" (Physician \# 6).

\section{Poor resource allocation.}

Moreover, the study showed inadequate of resources. Both human and non-human resources are among the ethical challenges in the ICU. The decisions of allocating resources in ICU have reported to be a challenging among healthcare workers. This concern was expressed below by two quotes from two participants.

"Here in Bugando, decisions are very challenging, because the ICU is not well established we have some materials but actually are not enough" (Nurse \# 8).

"The availability of beds is a challenge, we need to have more beds but for only present some time is not enough. It is challenging once you receive a patient who needs ICU care and that time all bed are fully covered" (Nurse \# 9).

\section{Limited access of ICU care.}

The study had revealed that, The ICU infrastructure of Bugando Medical Centre limit the access of care to other patients. The ICU room is small and occupies a limited number of beds. Bugando Medical Centre has only one AICU which is used to all hospital departments and all referral patients from eight regions of Lake Zones. Therefore some time demand for ICU care for patients is high but the ICU does not able to accommodate all patients at a time. These limit the access of other patient to ICU, however they also making the healthcare workers in an ethical dilemma to decide who should be given priority to ICU care. As one of the participant responded. 13

"...The ICU needs more beds but this should relate with room itself, the room we have is enough for only twelve beds. If we could have enough space also we could need more bed" (Nurse \# 9).

\section{Shortage of human resources}

The study showed a shortage of human resources in ICU; the available human resource is 26 nurses and two physicians and one registrar, who are permanent employees in the department. Human resource capacity in the ICU of Bugando Medical Centre is not enough; one nurse can care more than three to four 
patients per shift. These sometimes affect the quality of care for patients. ICU patients always need special and close attention. The nurse patient ratio should be at least one nurse for two patients in order to provide a good quality care. However for the Bugando ICU is different. This is discussed below by several participants.

"... although we know that, one patient should be cared by one nurse but here is vice versa one nurse care three to four patients who in is critically ill. So this is a big challenge, may be the administrator may think about this, so that the care which are delivered to be goo and quality" (Nurse \# 12).

To improve quality of care in the ICU of Bugando Medical Centre, the hospital management should increase the number of staff including nurses, physicians and intensivist.

"...for the staff we have a shortage, in order to provide good and quality care to our critically ill patient in ICU should at least [be] one nurse [to] two patients or if there is enough staff should [be] one [patient to nurse] ratio one, one nurse one patient but nurse's care [for] two, three up to four patients."(Nurse \# 10).

\section{Discussion}

This study has revealed that, there a variation in perspectives of healthcare workers towards withholding and withdrawing life support measures from terminally ill patients. However, the healthcare workers face many challenges in decision making to withhold and withdraw life support measures.

\section{Perception and Ethical challenges facing healthcare workers in making ethical decisions of withholding and withdrawing life support from terminally ill.}

The study aimed at exploring the perceptions and ethical challenges of healthcare workers on withholding and withdrawing life supportive measures from terminally ill patients. This study found that, healthcare workers have diverse thoughts and perceptions in the practice of withholding and withdrawing life support from terminally ill patients. Similar to the study conducted in Asia the decision on withholding and withdrawing life supportive measures varies according to economic status, cultural, religious, age, years in clinical practice, years in ICU and legal concerns. However, reported considerable variation in doctor's decision to forgo specific life sustaining treatments.

However, the findings has revealed that, Healthcare workers still are facing many challenges in making decision of withholding and withdrawing life support from terminally ill patients. The practice brought diverse views among healthcare workers, the study is similar to article written by Massimo stated, end of life is among the prominent area of controversial in contemporary bioethics. However, the absence of guidelines on when to consider withholding and withdrawing life support from terminally ill patients is one of the findings in this study. The healthcare workers lack guideline in withholding and withdrawing life support from terminally ill patients. This brings the issue of inconsistency in the practice. This 
situation is different from the Netherland, where they have provided the guideline for healthcare workers to consider in withholding and withdrawing life support which guides all medical team ${ }^{3}$.

In addition to unfair distribution of resources such as ICU beds, ICU infrastructure, mechanical ventilators and human resources in the ICU is among the factors that put healthcare workers in a difficult position for making decision on withholding and withdrawing life support.

The study has found that, there is a limited resource in ICU and patient stay until the nature takes place. However some healthcare workers opinions were to consider withholding or withdrawing life sustaining therapies for the irreversible patients. They perceived to continue to use resources for the patient, who has no hope of recovery, is wastage of money and materials. However, the study is similar to Lanken et al,stated continuing to use resources for the hopeless patient is viewing as improper rationing and misuse of resources.

The study reported a high demand of ICU care, as compared to the capacity of the ICU. Often times the ICU demand is high but the beds are few, this limit accessibility of other patients to ICU care. The study conducted by stated that, "the prolongation of life for the patient who had proved the life is irreversible is ethically unacceptable." The risks and benefits of further treatment should be analyzed in order to save finite resources. Due to finite resources in the ICU, healthcare workers have established mechanisms to ensure that resources are distributed fairly. The study revealed, patients are ranked according to their level of seriousness. Those who are at least recovery are transferred to the normal ward. Comparable to Simon, said that, "the issue of rationing is a common ethical concern in Tanzania." Therefore rationing is inevitable in an area with scarce resources.

\section{Conclusion}

The practice of withholding and withdrawing life support measures from terminally ill patients in ICU of Bugando Medical Centre face many challenges. However, there is considerable factors which believed by health care workers to withhold or withdrawing life support measures from terminally ill patients. Due to lack of legal and ethical framework to guide the practices, physicians and nurses feared to make ethical decision.

\section{Recommendations}

i. The government and stakeholders should look forward the establishment of legal and ethical framework in the practice of withholding and withdrawing life support measures from terminally ill patients in ICU.

ii. The hospital administration should look forward to establishment mechanism of guidance and dealing with ethical dilemmas in the ICU as well as to other clinical settings.

iii. Further study can be done to determine underlying issues relating to end of life ethical issues and magnitude of the problem in ICU囚s of Tanzania. 


\section{Abbreviations}

AICU Adult Intensive Care Unit

BMC Bugando Medical Centre

CUHAS Catholic University of Health and Allied Sciences

DMRET Dartmouth MUHAS Research Ethics Committee

ICU Intensive care unit

\section{Declarations}

\section{Ethics approval and consent to participate.}

The nature of the consent was written consent. The consent was explained the risks and benefits of the study. Participants were voluntarily participated in the study.

The study was approved by two ethical committee namely MUHAS ETHICAL COMMITTEE with Reference number DA.287/298/01A/ and CUHAS/BMC RESERCH ETHICAL COMMITTEE (CREC) CERTIFICATE NUMBER CREC 351/2019.

Consent for publication.

\section{N/A}

Availability of data material.

All data generated or analyzed during this study are available from corresponding author on reasonable request.

\section{Competing interest}

The author(s) declare(s) that they have no competing interests.

\section{Funding.}

The study was funded by DARTMOUTH RESERCH ETHICS PROGRAM (DMRET, GRANT NUMBER 5R25TW007693-08 from proposal designing up to the completion of the study.

\section{Authors contributions.}

NG: The corresponding author develops the proposal, collects and analyzed the data.

PR: revised the concept note, advice and made changes in the development of full proposal. 
Acknowledgement:

I wish to acknowledge the following to the success of this report

First, Dr Renatha Joseph head of department of Bioethics and Health professionalism, Muhimbili University of Health and Allied Sciences, Daima Bukini Assistant Lecturer Muhimbili University of Health and Allied Sciences, and Prof Muhsin Abood principal Investigator, DARTMOUTH MUHAS Research Ethics Program (DMRET).

\section{References}

Beichlin K etal. (2014 (9)). The Ethics of Withholding and Withdrawing Medical treatment . Multidisplinary Respiratory Medicine, 39.

De Graef. N, Savelkoul. C, Kompanje. E, \& Tjan. D,. (2016). End of life practice: Ensuaring quality of dying during withdrwal of life sustaining measures. Journal of critical Care, 24(4).

Hinka etal. (2002). Factors affecting Physcians decisions to Forgo Ife sustaining treatments in terminal care. . Journal of Medical Ethics (28), 109-114.

Iqbar. S. (2013(4)). Accepting end of life Care Realities - When choice are limited. Journal of Critical Research Bioethics, 4.

Lanken. P, etal. (1997 (156)). Fair Allocation of Intensive Care Unit Resources. American journal of Respiratory Critical Care Medicine, 1282-1301.

Lobos, M. De Simon. F, Jacob. S, Estella, A etal. (2017). Decision Making on Withholding or Withdrawing Life Support in the ICU. A World Perspectives. . CHEST. 152 (2) , 321-329.

Luchet. M. (2013 (6)). Intensive Care Resources Allocation: When difficult choices have to be made. British Journal of Medical Practitioner , 4.

M, S. (2015). Resource Allocation for Health in Tanzania- Determinants and Development Implications. Retrieved March 23, 2018, from http://hss.ulb. uni-bonn.de/2015/3966/3966/.pdf

McCarthy. J, C. L. (2016). Palliative Care for the person with Dementia. Document 6: Ethical Decisions Making in End of Life Care and the Person with Dementia. Dublin: Irish Hospice Foundation.

Phua etal. (2015). Withholding and Withdrawing Life support from Intensive care Unit. JAMA Intern Med, 363-371.

\section{Figures}


E thical challenges in making ethicaldecision of withholding and withdrawing life support measures from terminally ill.

- Dilemma in decision making

- Res ource allocation

- Limited access toICU care

- Shortage of staff
F actors influences ethical decision making in withholding and withdrawing life support measures

- Patient prognosis

- Legal concerns

- Family decisions

- Professional ethics \& obligations

- Individual cultural \& religious beliefs.

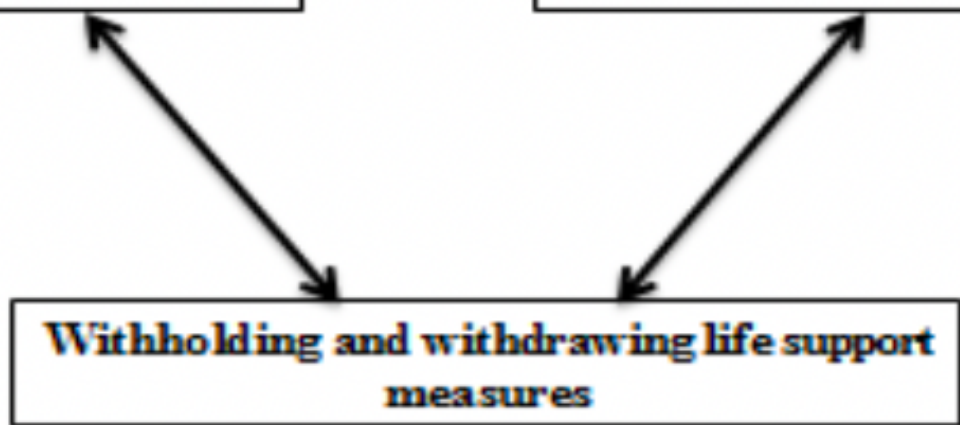

Figure 1 Summary findings illustrating how the two categories are related to one another and subcategories within each category.

Figure 1 